\title{
Novel Method for Surface Tension Measurement: the Drop-Bounce Method
}

\author{
Yifan Sun $^{1}\left[\right.$ D Hiroaki Muta ${ }^{1} \cdot$ Yuji Ohishi $^{1}$
}

Received: 18 December 2020 / Accepted: 15 April 2021 / Published online: 26 April 2021

(c) The Author(s) 2021

\begin{abstract}
The surface tension of liquids at high temperatures is generally measured with the well-established oscillating drop method in a contactless environment. However, technical difficulties in surface tension measurements make it hard to apply the oscillating drop method to the aerodynamic levitation (ADL) system, the most reliable levitation technique for liquids with low electrical conductivity. In this study, we developed a novel drop-bounce method that can be used within an ADL system to measure the surface tension of liquids. A levitated molten sample was first dropped onto an inert substrate through a splittable nozzle. The rebounded sample's oscillatory motion behaved as it would under microgravity conditions during its free-fall, and oscillations were obtained only in the $1=2, m=0$ mode. Fourier transformation of the oscillation pattern provided resonant frequency of the $1=2, m=0$ mode and enabled the calculation of the surface tension of the sample under knowledge of its mass. Furthermore, a short experimental duration of less than $50 \mathrm{~ms}$ significantly reduced the possibility of surface evaporation in the sample. Our measured surface tension data from $1354 \mathrm{~K}$ to $1827 \mathrm{~K}$ for gold exhibited a standard deviation of $13.4 \mathrm{~mJ} / \mathrm{m}^{2}$ and were consistent with the data published by Egry et al. under microgravity conditions, with a maximum deviation of $1.5 \%$ between the two fitted linear equations.
\end{abstract}

Keywords Aerodynamic levitation $\cdot$ Liquid metals $\cdot$ Surface tension $\cdot$ Gold

\section{Introduction}

The thermophysical properties of liquid metals and their oxides, such as density, surface tension, and viscosity, are significant for industrial applications of these materials and for understanding the structure and order within these liquids. Although the high melting temperatures of liquid metals pose a challenge for contact experiments, the development of levitation technologies, such as electrostatic levitation (ESL) (Paradis et al. 2001; Rhim et al. 1999; Ishikawa et al. 2001), electromagnetic levitation (EML) (Egry et al. 1995; Bayazitoglu et al. 1998; Seidel 2011; Sauerland et al. 1993), and aerodynamic levitation (ADL) (Kargl et al. 2015; Langstaff et al. 2013; Benmore and Weber 2017; Kondo et al. 2019), over the past years has facilitated measurements at high temperatures without contamination. The suitable material for a specific type of levitation technique depends on the operating principle of the levitator.

Yifan Sun

yifansun2016@ms.see.eng.osaka-u.ac.jp

1 Graduate School of Engineering, Osaka University, Osaka 565-0871, Japan
Generally, oxides are suitable for the ADL system, and metals are suitable for the ESL or EML systems.

With respect to the three thermophysical properties discussed earlier, density can be determined by evaluating the image of the levitated drop using all three techniques, i.e., ESL, EML, and ADL. In contrast, measuring the surface tension and viscosity by the oscillating drop method (Rhim et al. 1999; Egry et al. 2005) is significantly difficult. Rayleigh's law (William 1997) directly relates the surface tension to the mass and resonant frequency of a spherical, non-rotating sample. However, under normal gravity, i.e. non-force-free, conditions, the resonant frequency of the $1=2$ mode (Rayleigh frequency) can split into up to five peaks due to sample's rotation and non-spherical shape (Busse 1984). For EML, a sum rule (Cummings and Blackburn 1991) based on the electromagnetic force distribution on the levitated sample was introduced to mathematically relate the resonant frequencies of the five $1=2$ mode observed under normal gravity to the resonant frequency of the single $1=2$ mode obtained under microgravity. Egry et al. (1995) performed EML experiments under both normal and microgravity conditions and confirmed 
that applying such a sum rule is valid. However, a sum rule does not exist for ADL. Langstaff et al. (2013) performed ADL experiments under normal gravity conditions and attempted to calculate the surface tension by applying the same correcting terms that are applied for EML. They concluded that previous oscillating drop studies performed with ADL using only the resonace frequency of the $1=2$, $\mathrm{m}=0$ mode could have underestimated the surface tension by approximately $20 \%$. However, the validity of applying the same sum rule for EML and ADL is debatable. Hakamada et al. (2017) recently showed that through a well-controlled experiment, a natural surface oscillation of the $1=2, m=0$ mode can be induced and one might not need a sum rule for surface tension calculations. Given the difficulty of ground-based experiments, Matsumoto et al. (2005) eliminated the shifting of the $1=2$ mode peaks by allowing an electromagnetically levitated sample to oscillate during a free-fall and successfully determined the surface tension of liquid copper. However, as the method demonstrated by Matsumoto et al. is based on EML, it is not suitable for measuring the surface tension for materials with low electrical conductivity.

Besides the contactless methods mentioned above, contact methods such as the pendant drop (Stauffer 1965; Man 2000; Vinet et al. 1993; Egry et al. 2010), sessile drop (Egry et al. 2010; Ricci and Novakovic 2001; Sageman 1972), and maximum bubble pressure method (Simon 1851) have been used to determine the surface tension of molten metal systems. The pendant drop and sessile drop methods were combined together to investigate the surface tension of $\gamma$-TiAlbased alloys at temperatures as high as $2400 \mathrm{~K}$ (Nowak et al. 2010). However, the disadvantages of these contact methods become quickly apparent. As the measurement temperature approaches and surpasses $3000 \mathrm{~K}$, it becomes increasingly difficult to maintain a homogeneous temperature profile and to select appropriate materials to be used as supports or needles to reduce contamination.

It is difficult to obtain accurate surface tension data using the oscillating drop method with ADL under normal gravity conditions. Therefore, we introduce a novel method that can potentially be used for ADL of both metals and oxides. In designing such a method, issues such as lack of a sum rule for calculation as well as long experiment duration that can lead to sample oxidation or evaporation must be addressed. Our drop-bounce method addresses these two major issues by allowing the aerodynamically levitated molten sample to drop a few millimeters onto a boron nitride platform beneath a splittable nozzle and bounce back up. During the rebound period, the sample is essentially free-falling, and its oscillation is excited without the presence of any external forces. Surface tension can be directly related to the resonant frequency of the oscillating sample, thereby eliminating the need for a sum rule for ADL. Furthermore, as the sample travels through a distance of 2-3 mm, an experiment time of less than $50 \mathrm{~ms}$ can significantly reduce the chance of surface evaporation or oxidation of the sample.

\section{Experimental Method}

\section{Structure of Aerodynamic Levitation System}

Detailed designs of the ADL systems have been extensively reported by Langstaff et al. (2013) and Kargl et al. (2015) ; only specific modifications made to their design for this study are reported here. An image and a simplified schematic of the ADL setup used in this study are shown in Figs. 1, 2. A $110 \mathrm{~W}$ fiber laser (976 nm, Pearl), which was mounted directly above the sample chamber, was used to heat and melt metal samples, and the temperature of the sample's surface was monitored and recorded using a pyrometer (1550 nm, IR-CAI8CN, CHINO) and a data logger (GL900, Graphtec), respectively. A longpass filter of $1200 \mathrm{~nm}$ (FELH1200, Thorlabs) was installed in front of the pyrometer to cut off any laser reflected from the surface of the sample, which heavily influences the recorded temperature. The fiber laser's output power was controlled using a WinVue user interface software and was cooled by a cooling water system (LTC-S300C, AS ONE). Two ports on top of the sample chamber allowed for direct observation of the sample with charge-coupled device (CCD) cameras. One of the ports was equipped with two shortpass filters at $550 \mathrm{~nm}$ (FES0550, Thorlabs) and $950 \mathrm{~nm}$ (FESH0950, Thorlabs) to observe the levitated molten sample. The other port was equipped with a single shortpass filter at $950 \mathrm{~nm}$ (FESH0950, Thorlabs), which was mainly used to observe the sample at lower temperatures. An additional port was used to provide lighting to illuminate the sample chamber.

Two ports were made available on the two sides of the sample chamber to record shadowgraph images (Ishikawa et al. 2001) of the free-falling molten sample during the experiment. A white light source (SLA-100B, OptoSigma) with a $450 \mathrm{~nm}$ bandpass filter (FBH450-10, Thorlabs) and a high-speed camera (MEMRECAM HX-7s) with a 450 $\mathrm{nm}$ bandpass filter (FBH450-10, Thorlabs) and a telecentric lens $(1.0 \times, 2 / 3$ ", Gold TL, Edmund) were used to record the experiment at a frame rate of $4000 \mathrm{fps}$, a resolution of $1024 \times 928$, and a shutter speed of $10 \mu \mathrm{s}$.

A simplified schematic of the alumina sample chamber is also shown in Fig. 2. A rectangular-shaped aluminum shutter was installed above the nozzle to block the top laser beam temporarily, if required. A system containing a solenoid valve, linear motor, and argon gas controlled the opening and closing of the shutter. To drop the levitated sample during the experiment, we designed a conical 
Fig. 1 A setup of the ADL system used in this study

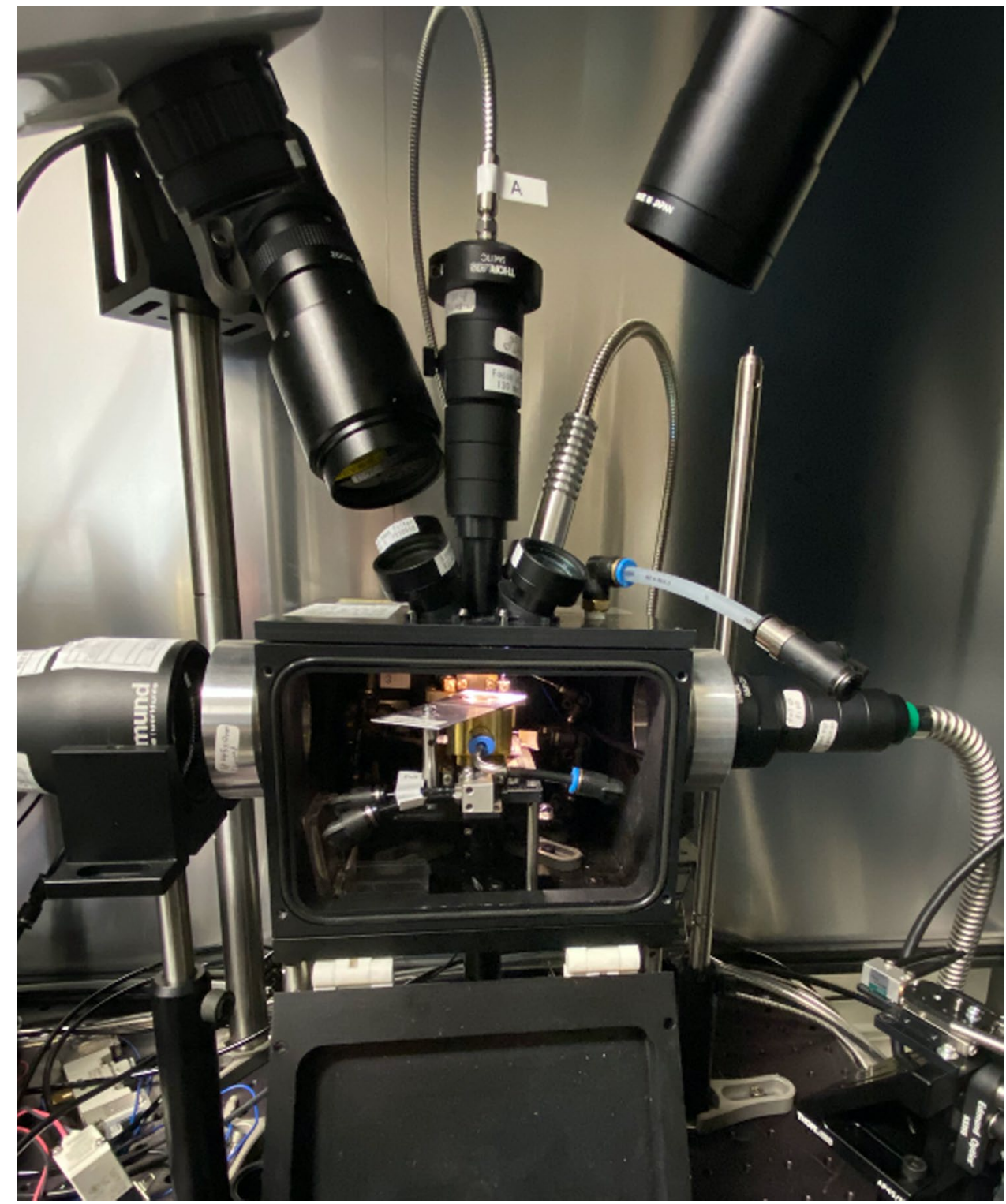

nozzle ( $1 \mathrm{~mm}$ diameter) that can split into two parts. This operation was also controlled by a system of solenoid valves, linear motors, and gas supply, as shown in Fig. 3.

The solenoid valves were connected to allow the shutter to function independently (blocking/unblocking the laser beam) or simultaneously (blocking the laser beam and splitting the nozzle, and vice versa) with the nozzle. The levitation gas, without being preheated to match the temperature of the levitated molten sample, was supplied through a hollowed section within one side of the nozzle. A thermocouple was used to monitor the nozzle's surface temperature to prevent the nozzle from overheating and damaging the gas supply tubes. Located approximately 3 $\mathrm{mm}$ underneath the splittable nozzle was a stainless-steel rod with a boron nitride plate that was used as the platform for molten samples to bounce off from.

\section{Surface Tension Analysis: the Drop-bounce Method}

The surface tension of an oscillating sphere is related to its resonant frequencies through Rayleigh's equation (William 1997),

$$
\gamma=\frac{3 \pi v_{R}^{2} M}{l(l-1)(l+2)}
$$

where $\gamma$ is the surface tension, $v_{R}$ is the resonant frequency of a specific oscillation mode, $M$ is the mass of the oscillating sphere, and $l$ is the oscillation mode. In general, the $1=2$ mode is the most dominant oscillation mode ( $1=1$ being translational motion) and can be easily recognized by the naked eye. However, for all levitation experiments conducted under normal gravity conditions, some 


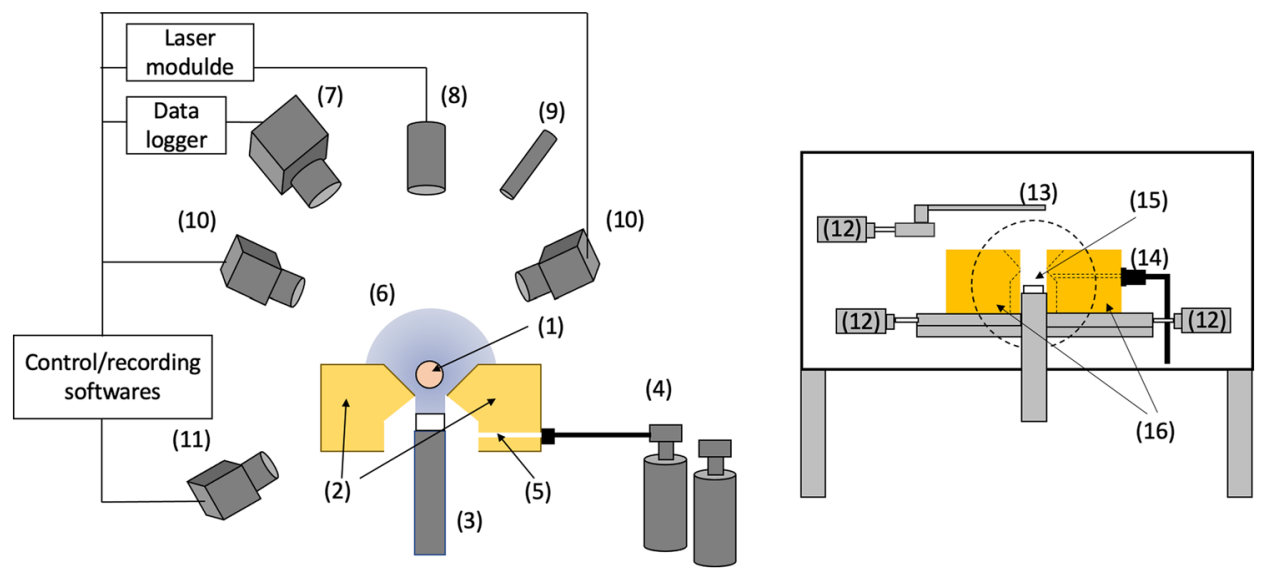

Fig. 2 Schematic of the ADL system used in this study. (1) Levitated sample position; (2) splittable nozzles; (3) stainless-steel supporting rod with a boron nitride plate glued on top, positioned at approximately $3 \mathrm{~mm}$ beneath the nozzle; (4) gas supply for levitation and for driving the nozzles; (5) levitation gas passageway within the nozzle, (6) background light (with a $450 \mathrm{~nm}$ bandpass filter); (7) a pyrom-

external forces (electrostatic, electromagnetic, or inert gas) are used for levitation; using external forces can split the $\mathrm{l}=2$ mode into five modes $(\mathrm{l}=2, \mathrm{~m}=0, \pm 1, \pm 2)$ as the sample is no longer perfectly spherical and rotational motion can occur (Busse 1984). To continue using Eq. 1, a sum rule (Cummings and Blackburn 1991) is necessary to combine the resonant frequencies of the five modes. However, as mentioned in the introduction section, unlike the electromagnetic force used in the EML, the force of levitation gas applied to the sample in ADL is very complicated and no sum rule specific to the ADL system is currently available (Langstaff et al. 2013).

The drop-bounce method developed in this study eliminates the need for an external force to induce oscillation in the molten sample such that the $1=2$ mode is not split and the sample behaves as if under a microgravity environment. To achieve force-free oscillation, after the molten sample is stably levitated, the nozzle splits, and the spherical sample drops onto the boron nitride platform that is placed eter; (8) a $110 \mathrm{~W}$ fiber laser operating at $976 \mathrm{~nm}$; (9) white light to illuminate the sample chamber; (10) observation-use CCD cameras; (11) high-speed camera used for shadowgraph recording. The chamber consists of (12) gas-driven linear motors, (13) laser beam shutter, (14) route of the levitation gas, (15) boron nitride platform, and (16) splittable nozzles

a few millimeters beneath the nozzle. Upon contacting the boron nitride platform, the spherical sample reaches maximum deformation and then bounces back up. Oscillation is then induced as the surface tension of the deformed sample minimizes its surface area and retains its original spherical shape. To further explain this process, time-lapse images of the oscillating sample are shown in Fig. 4. In the absence of any other oscillation modes, a single $\mathrm{l}=2, \mathrm{~m}=0$ oscillation mode can be easily recognized during the later stages of the rebound period. We were able to induce a single $1=2, m=0$ oscillation mode with passing time as the shape of the sample at maximum deformation $(14.3 \mathrm{~ms})$ was similar to that of an oblate spheroid of the $1=2, m=0$ oscillation mode. Because the shape of the sample was not a perfect oblate spheroid, higher-order oscillations were present at much smaller intensities as the sample bounced upward, which can be observed in the picture taken at 19.5 ms. As the droplet continued to oscillate, the sample's viscosity led to a continuous decrease in its energy, thereby
Fig. 3 Laser beam shutter and splittable nozzle setup. On the left, the shutter does not block the laser beam and the splittable nozzle is shut, allowing heating and levitation. On the right, the shutter blocks the laser beam and the splittable nozzle is split, allowing the sample to drop
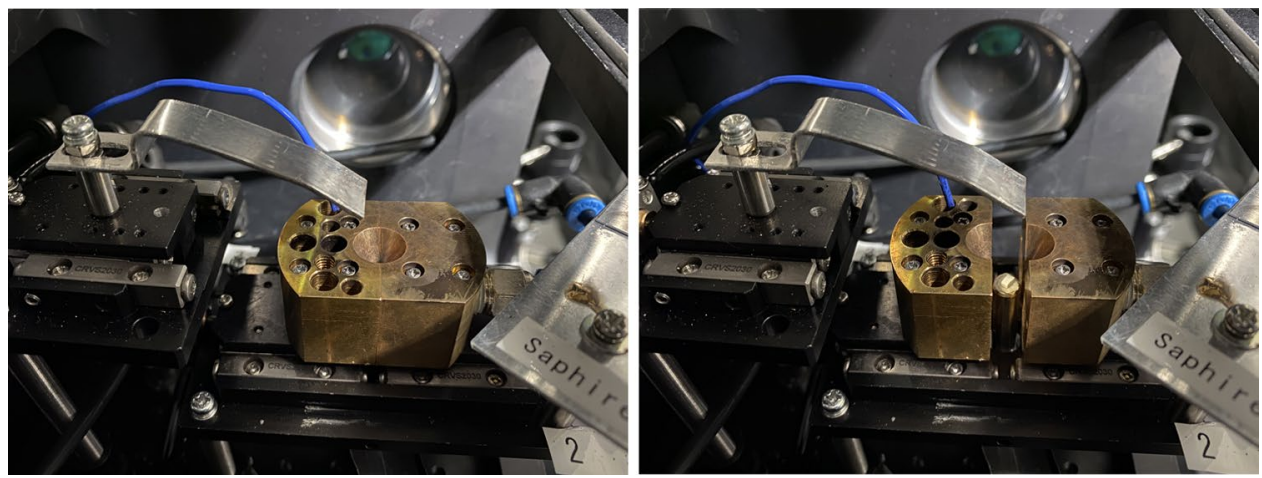
Fig. 4 Time-lapse images of molten gold sample during drop-bounce surface tension measurement. At $6.0 \mathrm{~ms}$, the spherical sample approached the boron nitride platform. At $14.3 \mathrm{~ms}$, maximum deformation was achieved. At $19.5 \mathrm{~ms}$, the sample deformation remained sufficiently large to be used for oscillation analysis. From $26.3 \mathrm{~ms}$ onward, a single $\mathrm{l}=2$, $\mathrm{m}=0$ mode oscillation can be observed
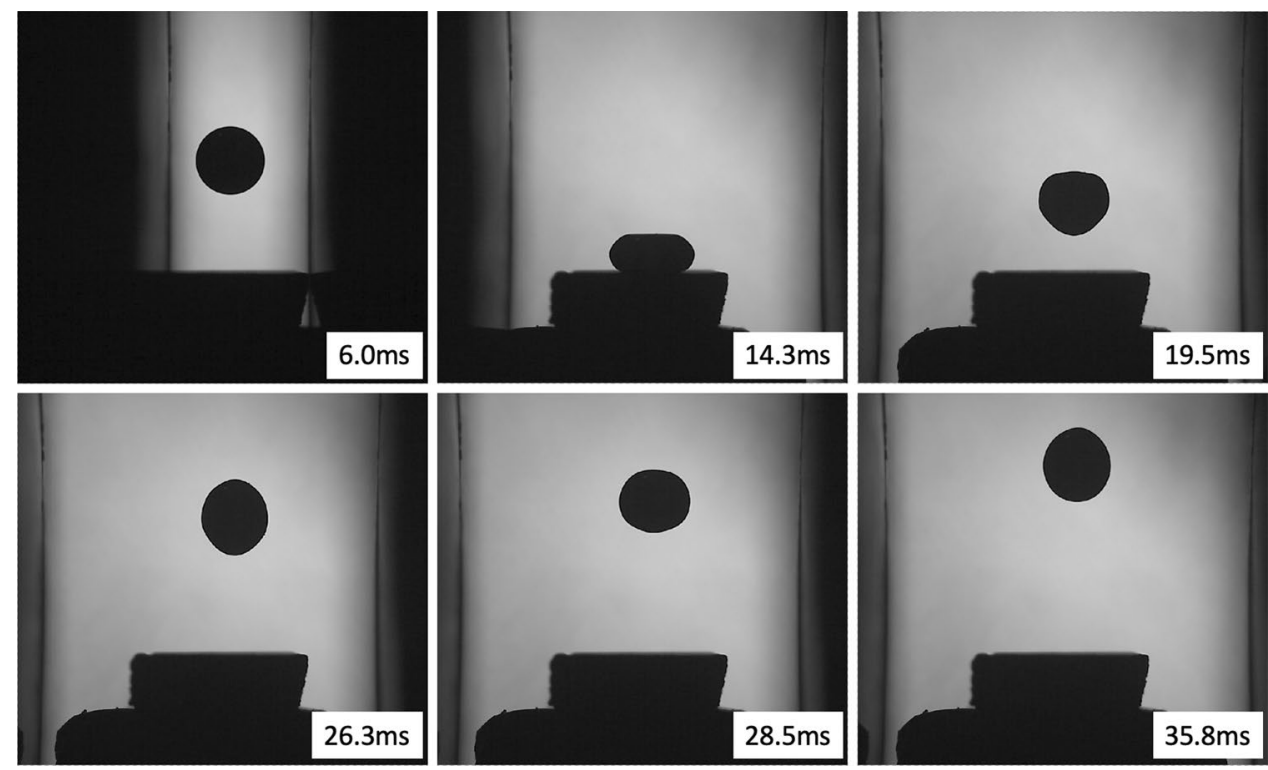

resulting in a decrease in the oscillation intensities across all oscillation modes. As the $\mathrm{l}=2, \mathrm{~m}=0$ mode had the highest intensity, it was the last to disappear. This explains why the sample oscillation became significantly more regular (into an $\mathrm{l}=2, \mathrm{~m}=0$ mode fashion) from $19.5 \mathrm{~ms}$ to $35.8 \mathrm{~ms}$. For materials with extremely low viscosity values, a longer duration might be required for the higher-order oscillations to disappear completely, and the dropped distance should be adjusted accordingly. The $1=2, \mathrm{~m}=0$ oscillation mode can be confirmed by Fourier transformation of the sample's oscillation pattern shown in Section 2.4. Through the drop-bounce method, it is then possible to obtain the surface tension without a sum rule using Eq. 2 by directly locating the resonant frequency of the single $1=2, m=0$ oscillation mode.

$\gamma=\frac{3}{8} \pi v_{R}^{2} M$

Another advantage of the drop-bounce method over the conventional oscillating drop technique in determining the surface tension of liquids is its significantly shorter experimental duration. For liquids with high vapor pressure, the drop-bounce method allows the measurements to be completed within $50 \mathrm{~ms}$ to significantly reduce the possibility of evaporation, which can heavily influence the surface properties of the liquids. However, as the sample will touch the boron nitride platform during the process, the surface tension of undercooled liquids cannot be measured with the current set-up. Depending on the sample's reactivity, the material used as the bounce platform should be carefully chosen to minimize the amount of potential contamination.

\section{Temperature Correction}

As mentioned previously in Section 2.1, the temperature of the sample was monitored using a pyrometer, and the data logger recorded the temperature every $1 \mathrm{~ms}$. As the pyrometer treated the sample as a black body, the as-recorded temperature must be re-calibrated using Wien's law:

$\frac{1}{T}-\frac{1}{T_{P}}=\frac{1}{T_{L}}-\frac{1}{T_{P, L}}$

Here, $T$ is the re-calibrated temperature, $T_{P}$ is the temperature recorded by the pyrometer, $T_{L}$ is the liquidus temperature (1337K for gold (Hieu and Ha 2013)), and $T_{P, L}$ is the temperature recorded by the pyrometer at the melting point. On the cooling curve, $T_{P, L}$ can be identified by the sharp rise in temperature at the end of undercooling. To apply this correction to temperatures higher than the melting point, we assumed that the emissivity of each liquid sample at a specific wavelength is independent of temperature and is equal to the emissivity value at the melting point (Krishnan et al. 1990). An example of the temperature curve before and after re-calibration is shown in Fig. 5.

During the drop and bounce periods in the surface tension analysis, it was no longer possible to focus the pyrometer on the moving sample. To estimate the temperature of the bouncing sample, the value of the cooling rate is required. This was achieved by fitting the free-cooling part of the temperature curve with a quadratic equation against time, which allowed us to estimate the temperature of the sample during the drop and bounce phases. For clarity, an example of a fitted free-cooling curve is shown in Fig. 6. 


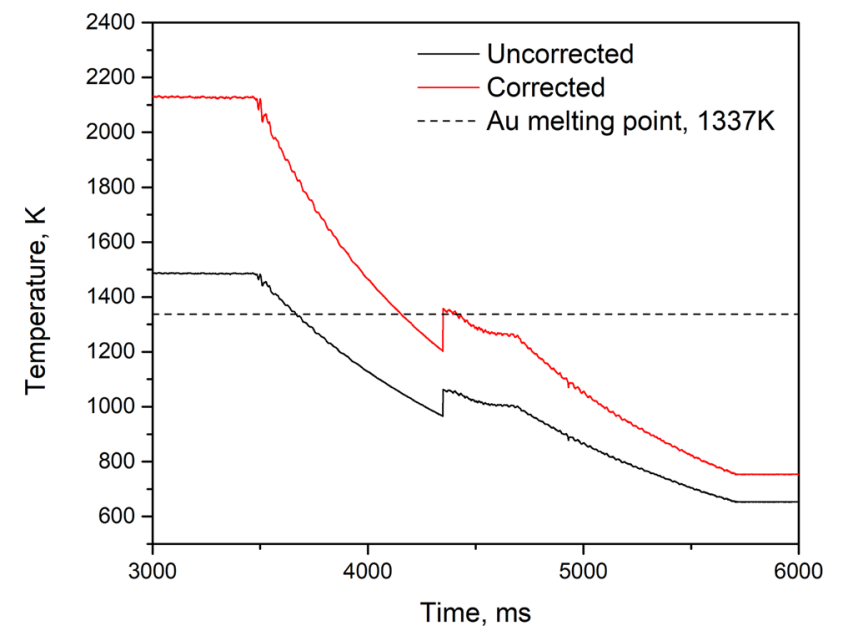

Fig. 5 Unadjusted (blackbody) and adjusted temperature curves with emissivity calculated by matching the temperature at the melting point

The experimental cooling curve shown in Fig. 6 was obtained while the sample was being levitated, and the surface area of a stably levitated sample is different from that of an oscillating sample. Based on our experimental data, the samples experienced approximately $0-10 \%$ changes in their surface areas during oscillation. Thus, an average change of $5 \%$ in the sample surface areas was considered to calculate the radiative heat loss during oscillation. The cooling curves of a sample that only experienced radiative heat loss during levitation and oscillation were calculated by assuming a constant surrounding temperature of $298 \mathrm{~K}$ using the Stefan-Boltzmann law; the cooling curves are shown in Fig. 7. A temperature difference of $1 \mathrm{~K}$ at $50 \mathrm{~ms}$ was observed, which is generally the maximum experiment time. However, by comparing the cooling curves obtained

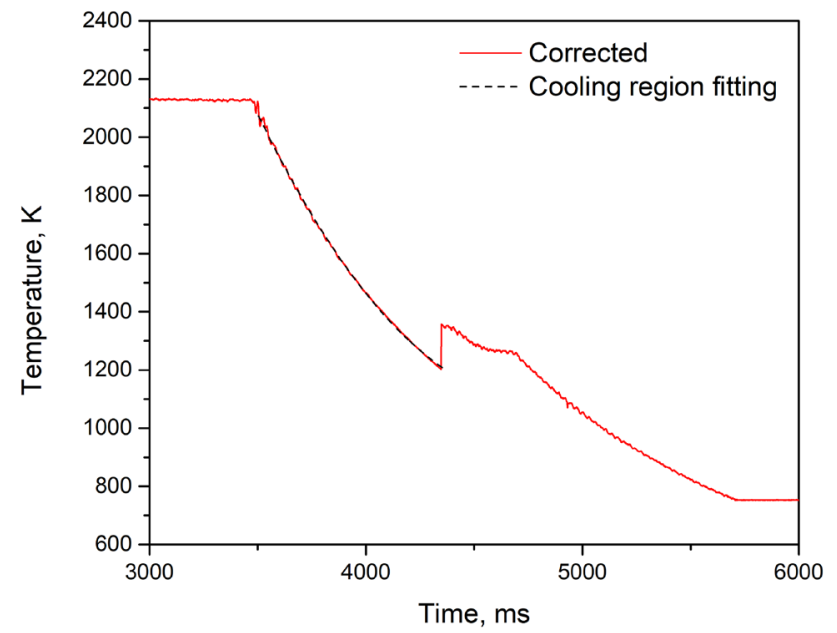

Fig. 6 Cooling behavior fitted with a quadratic equation

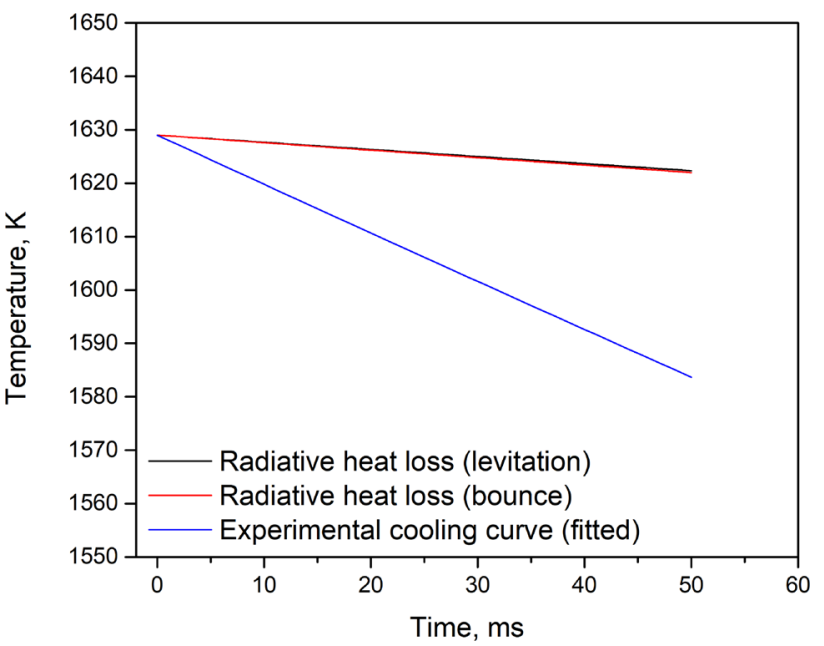

Fig. 7 Comparison between the calculated cooling curves by only considering the sample's radiative heat loss and the fitted experimental cooling curve

by considering only the radiative heat loss to the actual experimental cooling curve, we observed that, as expected, convective heat transfer to the surrounding Ar gas also heavily contributed to the sample cooling. Depending on the initial temperature at the drop, after $50 \mathrm{~ms}$ of experiment time, the temperature deviation caused by ignoring the convective heat loss was between $20 \mathrm{~K}$ and $50 \mathrm{~K}$. This indicates that using the fitted experimental cooling curve to estimate the temperature of the oscillating sample can result into a maximum temperature underestimation of $20-50 \mathrm{~K}$. This maximum deviation in temperature is only achieved when the convective heat loss is zero during sample oscillation, which is highly unlikely. Finally, we considered that the conductive heat transfer from the sample to the boron nitride plate is negligible since the maximum contact area between the sample and the boron nitride plate was less than $1 \mathrm{~mm}^{2}$, for an extremely short contact time around $0.5-1.0 \mathrm{~ms}$. Additionally, simulations performed on the heat transfer across solid-liquid interfaces have shown that the thermal boundary resistance exponentially increases with decreasing solid-liquid interactions (Xue et al. 2003; Giri et al. 2016). The liquid gold droplet displayed poor wetting behavior with the boron nitride platform; thus, we assumed that the heat loss through heat conduction was negligible.

As we evaluated the surface tension based on a series of images, the surface tension was not calculated for a specific temperature; however, it should be considered as an averaged value over a small temperature range. For the experiments conducted in this study, this temperature range was measured to be between $\pm 10 \mathrm{~K}$ and $\pm 20 \mathrm{~K}$. This small temperature range, in addition to the temperature 


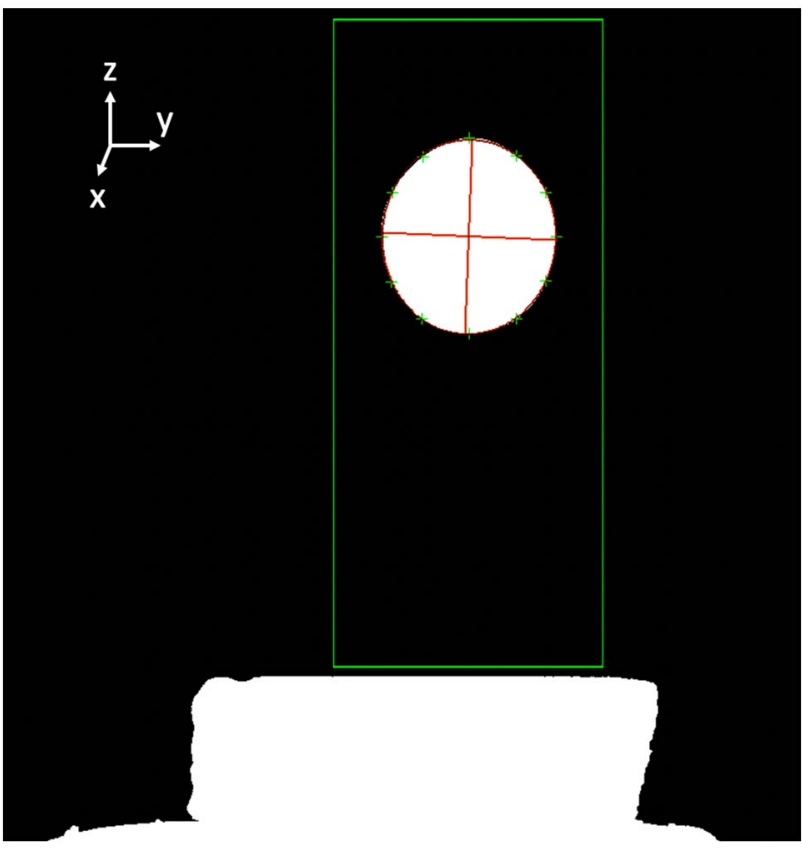

Fig. 8 Elliptical fitting of shadowgraph images using DIPP-Macro II

underestimation from using the fitted experimental cooling curves $(20-50 \mathrm{~K})$ to estimate the cooling rates of the samples, make up the total uncertainty in our measured temperature data. The calculated total uncertainty values in the estimated temperature specific to each of the samples are presented in Fig. 12, which is discussed later in the results and discussion section.

\section{Image and Data Analysis}

As mentioned in Section 2.1, shadowgraph images of the sample were taken at a rate of $4000 \mathrm{fps}$, a resolution of $1024 \times 928$, and a shutter speed of $10 \mu \mathrm{s}$. Elliptical fitting was then applied to these shadowgraph images by DIPPMacro II, an image analysis software (Fig. 8). Information on the major and minor axes of the oscillating sample can be extracted from the elliptical fitting to plot an oscillation pattern. With the high-speed camera setup, we can easily notice any unwanted sample rotations along the $\mathrm{x}$ - and y-axes (Fig. 8). Although we cannot determine whether there is a rotation along the $\mathrm{z}$-axis, the rotation along this direction does not affect the observed shape and oscillation of an ellipsoid-shaped sample.

For data analysis, a Hamming window was applied to the oscillation data, and Fourier transformation was performed to reveal the resonant frequency of the $1=2, \mathrm{~m}=0$ mode, as shown in Figs. 9, 10. For a harmonically oscillating spheroid of a given mass, the $1=2, m=0$ mode always has a greater amplitude for the oscillation in the vertical direction than that for the oscillation in the horizontal direction, which generates

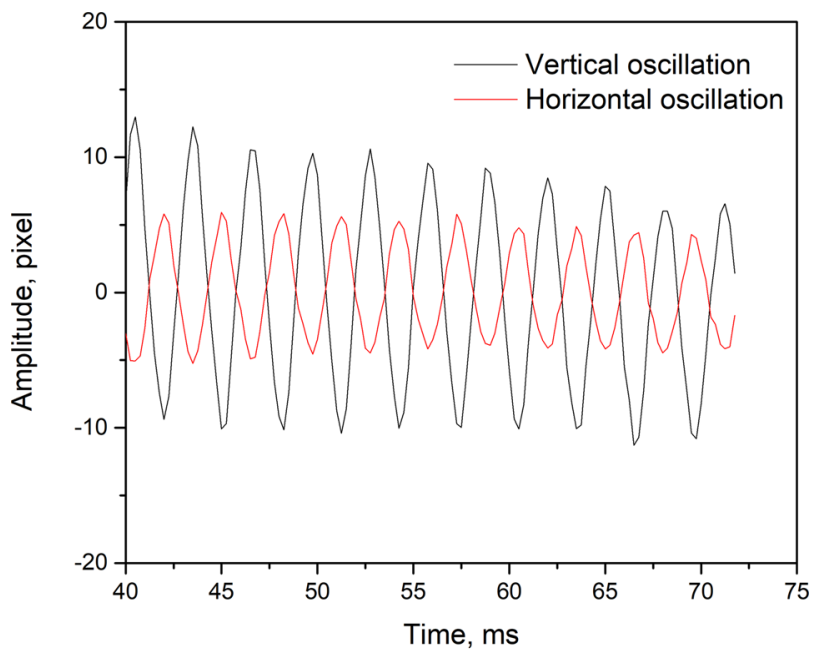

Fig. 9 Horizontal- and vertical-direction oscillation data extracted from elliptical fitting using DIPP-Macro II

sharper Fourier-transformed peaks. For this reason, Fourier analysis in this study was performed only on the vertical oscillation patterns, although the resonant frequencies obtained from the oscillations in both the directions should be the same. A Lorentzian fit was applied to the Fourier-transformed data to locate the frequency of the peak. We chose a Lorentzian fitting function because it describes the frequency domain of a damped harmonic oscillator. From Fig. 10, we can notice that the frequency resolution of the Fourier transformation is limited by the amount of data obtained within a short experiment time ( $\sim 50 \mathrm{~ms})$. The frequency spectrum's resolution can be further enhanced by increasing the time the sample spent

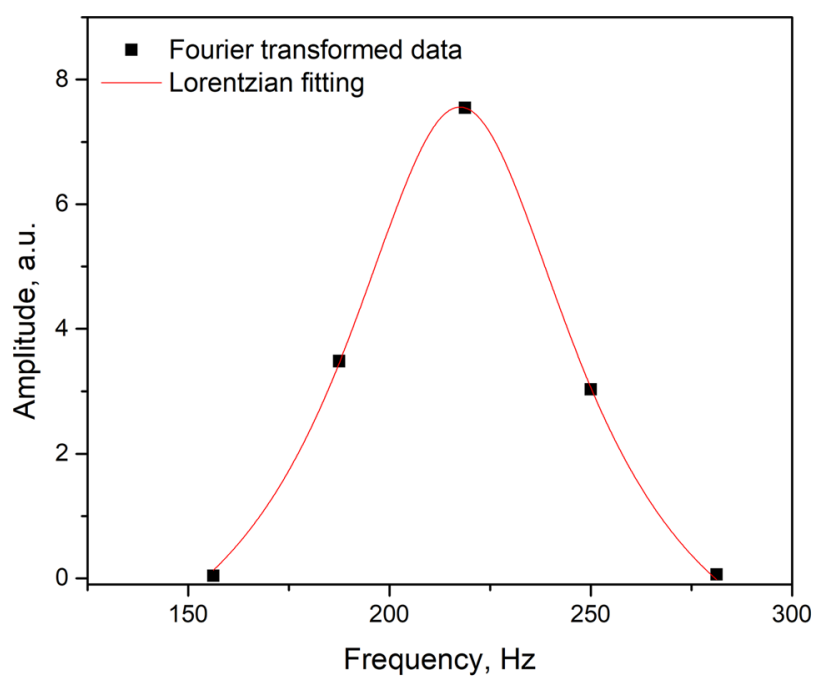

Fig. 10 A single resonant frequency peak for the $1=2, m=0$ mode obtained after Fourier transformation by applying Lorentzian function fitting 
oscillating mid-air, which will also further reduce the amplitude of potential higher order oscillations. In this study, the uncertainty resulted from the current frequency resolution is reflected in the standard error of the fitted peak of the Lorentzian function. Taking this into consideration during calculations gave us an uncertainty of about $1 \%$ in the reported surface tension data.

\section{Free-fall Verification}

The core assumption of the drop-bounce method is that the sample oscillates in the absence of external forces other than gravity, which ensures that the $1=2$ oscillation mode does not split into five peaks. To determine whether the sample was indeed undergoing a free-fall during the experiment, the acceleration of the sample needs to be calculated. The shadowgraphs of a stainless-steel ball with a known radius were taken before each experiment to convert pixels into millimeters. Of the eight gold samples used in this study, the average acceleration of the samples was $9.8 \mathrm{~m} / \mathrm{s}^{2}$ with a standard deviation of $0.1 \mathrm{~m} / \mathrm{s}^{2}$, which shows that air resistance was negligible. An example of the center of mass movement of a gold sample along the vertical z-axis of the shadowgraphs is shown in Fig. 11.

\section{Results and Discussion}

The gold samples used for surface tension measurements were cut from a gold wire of diameter $0.90 \mathrm{~mm}(99.95 \%$, AU-171474 Nilaco), and the masses of these samples were between $8.6 \mathrm{mg}$ and $19.1 \mathrm{mg}$. A constant flow of

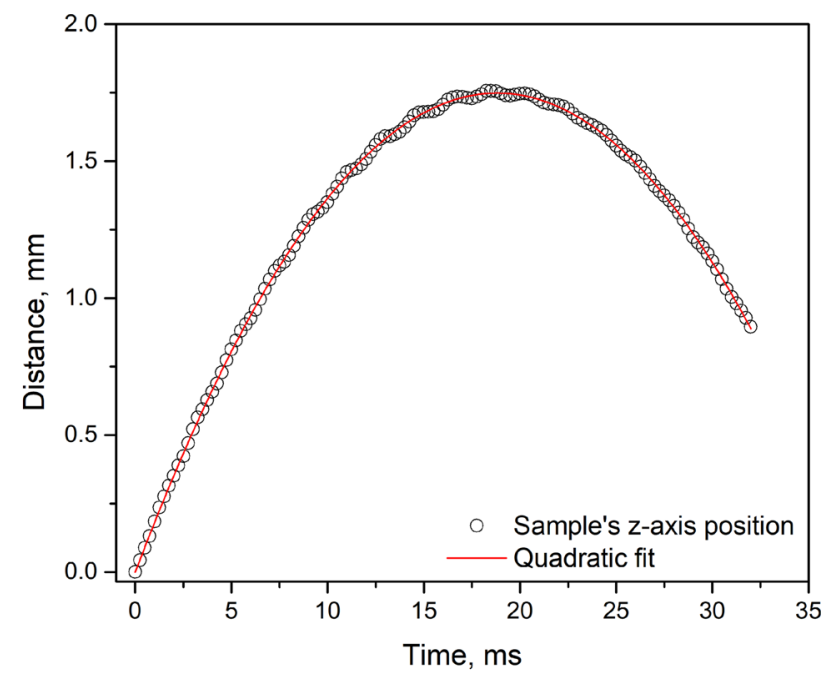

Fig. 11 Center of mass movement of a gold sample along the vertical $\mathrm{z}$-axis of the analyzed shadowgraph images, along with a quadratic fit for free-fall

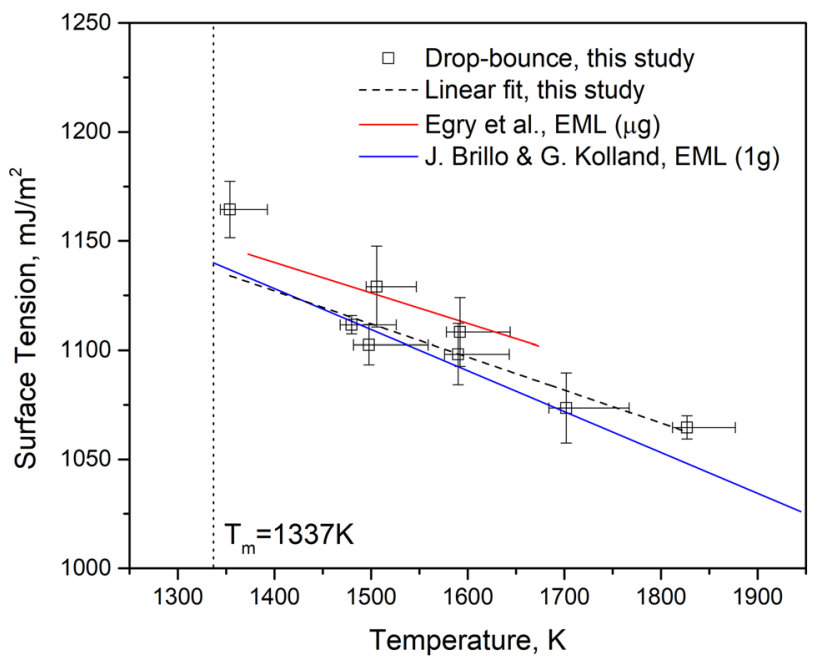

Fig. 12 Comparison of the surface tension of liquid gold with respect to temperature calculated by the drop-bounce method with the reference data

Ar gas was used to levitate the gold samples, and the molten samples were dropped at different temperatures. For each of the samples, 128 frames were captured to generate the oscillation pattern. The temperatures of the 128 data points can be calculated based on the estimated cooling rates described in Section 2.3. The surface tension data $\gamma\left(\mathrm{mJ} / \mathrm{m}^{2}\right)$ for liquid gold in the temperature range 1354-1827 K calculated by the drop-bounce method are shown in Fig. 12, and its temperature dependence can be expressed as:

$\gamma_{A u}=(1138 \pm 61)-(0.15 \pm 0.02) \times(T-1337)$

As shown in Fig. 12 and Table 1, our data have a standard deviation of $13.4 \mathrm{~mJ} / \mathrm{m}^{2}$, and are in good agreement with the reference data obtained using electromagnetic levitation (Egry et al. 1995; Brillo and Kolland 2016). The $r^{2}$ coefficient of our linearly fitted line is 0.87 . Notably, the fitting of our data is in good agreement (with a maximum deviation of $1.5 \%$ ) with that reported by Egry et al., who conducted the experiment under both normal and microgravity conditions. Although the

Table 1 Surface tension data of liquid gold measured by contactless methods and this study. For EML measurements, microgravity and normal gravity data were reported by Egry et al. and Brillo \& G. Kolland, respectively. Surface tension is expressed as $\gamma=\gamma_{L}+d \gamma / d T \times\left(T-T_{M}\right)$

\begin{tabular}{lll}
\hline Method & $\gamma_{L}$ & $d \gamma / d T$ \\
\hline Drop-bounce, ADL & $1138 \pm 61$ & $-0.15 \pm 0.02$ \\
Oscillating drop, $\mu$ g EML & 1149 & -0.14 \\
Oscillating drop, $1 \mathrm{~g}$ EML & $1140 \pm 60$ & $-0.183 \pm 0.04$ \\
\hline
\end{tabular}


linear relationship given by Egry et al. in Fig. 12 and Table 1 is for measurements conducted under normal gravity conditions, the data were corrected and found to be consistent with their data taken under microgravity. Thus, the primary goal of this study was achieved: we successfully replicated the ideal oscillation behavior of a sample under microgravity conditions on the ground for surface tension measurements. The drop-bounce method facilitates measurement of the surface tension with ADL without the need of a sum rule to relate the five shifted $l=2$ oscillation modes with surface tension. Most importantly, the drop-bounce method introduced here can be regarded as a complementary measurement method to the well-known oscillating drop method used in EML for surface tension measurements, particularly for samples with low electrical conductivity.

\section{Conclusion}

In this study, we developed a novel drop-bounce method for use in ADL systems for surface tension measurements. A splittable nozzle design allowed the molten sample to be dropped onto a boron nitride platform after a stable levitation was achieved. As the oscillation of the rebounding sample was free of external forces, the $1=2$ oscillation mode did not split into five modes $(\mathrm{l}=2, \mathrm{~m}=0, \pm 1, \pm 2)$, unlike its typical behavior for experiments conducted under normal gravity conditions. The linearly fitted surface tension equation for liquid gold obtained with the drop-bounce method in this study had a maximum deviation of $1.5 \%$ compared to the fitting performed by Egry et al. for experiments conducted under microgravity conditions. This small difference confirmed that the oscillation behavior of a sample under microgravity was successfully replicated by the drop-bounce method.

Acknowledgements This study is the result of the research on "Development of viscosity and surface tension measurement technique for molten core materials'; performed under the Nuclear Energy Science \& Technology and Human Resource Development Project (through concentrating wisdom) of the Japan Atomic Energy Agency, and it is supported by a Grant-in-Aid for the Japan Society for the Promotion of Science (JSPS) fellowship, [20J10376].

\section{Declarations}

Conflict of Interest The authors declare that they have no conflict of interest.

Open Access This article is licensed under a Creative Commons Attribution 4.0 International License, which permits use, sharing, adaptation, distribution and reproduction in any medium or format, as long as you give appropriate credit to the original author(s) and the source, provide a link to the Creative Commons licence, and indicate if changes were made. The images or other third party material in this article are included in the article's Creative Commons licence, unless indicated otherwise in a credit line to the material. If material is not included in the article's Creative Commons licence and your intended use is not permitted by statutory regulation or exceeds the permitted use, you will need to obtain permission directly from the copyright holder. To view a copy of this licence, visit http://creativecommons.org/licenses/by/4.0/.

\section{References}

Bayazitoglu, Y., Sathuvalli, U.B.R., Suryanarayana, P.V.R., Mitchell, G.F.: Determination of surface tension from the shape oscillations of an electromagnetically levitated droplet. Phys. Fluids 8 , 370 (1998)

Benmore, C.J., Weber, J.K.R.: Aerodynamic levitation, supercooled liquids and glass formation. Adv. Phys. X. 2, 717 (2017)

Busse, F.H.: Oscillations of a rotating liquid drop. J. Fluid Mech. 142, 1 (1984)

Brillo, J., Kolland, G.: Surface tension of liquid Al-Au binary alloys. J. Mater. Sci. 51, 4888 (2016)

Cummings, D.L., Blackburn, D.A.: Oscillations of magnetically levitated aspherical droplets. J. Fluid Mech. 224, 395 (1991)

Egry, I., Giffard, H., Schneider, S.: The oscillating drop technique revisited. Meas. Sci. Technol. 16, 426 (2005)

Egry, I., Lohoefer, G., Jacobs, G.: Surface tension of liquid metals: results from measurements on ground and in space. Phys. Rev. Lett. 75, 4043 (1995)

Egry, I., Ricci, E., Novakovic, R., Ozawa, S.: Surface tension of liquid metals and alloys - recent developments. Adv. Colloid Interface Sci. 159, 198 (2010)

Giri, A., Braun, J.L., Hopkins, P.E.: Implications of interfacial bond strength on the spectral contributions to thermal boundary conductance across solid, liquid, and gas interfaces: A molecular dynamics study. J. Phys. Chem. 120, 24847 (2016)

Hakamada, S., Nakamura, A., Watanabe, M., Kargl, F.: Surface oscillation phenomena of aerodynamically levitated molten al2o3. Int. J. Microgravity Sci. Appl. 34, 340403 (2017)

Hieu, H.K., Ha, N.N.: High pressure melting curves of silver, gold and copper. AIP Adv. 3, 112125 (2013)

Ishikawa, T., Paradis, P.F., Yoda, S.: New sample levitation initiation and imaging techniques for the processing of refractory metals with an electrostatic levitator furnace. Rev. Sci. Instrum. 72, 2490 (2001)

Langstaff, D., Gunn, M., Greaves, G.N., Marsing, A., Kargl, F.: Aerodynamic levitator furnace for measuring thermophysical properties of refractory liquids. Rev. Sci. Instrum. 84, 124901 (2013)

Kargl, F., Yuan, C., Greaves, G.N.: Aerodynamic levitation: thermophysical property measurements of liquid oxides. Int. J. Microgravity Sci. Appl. 32, (2015)

Kondo, T., Muta, H., Kurosaki, K., Kargl, F., Yamaji, A., Furuya, M., Ohishi, Y.: Density and viscosity of liquid $\mathrm{ZrO}_{2}$ measured by aerodynamic levitation technique. Heliyon. 5, (2019)

Krishnan, S., Hansen, G.P., Hauge, R.H., Margrave, J.L.: Emissivities and optical constants of electromagnetically levitated liquid metals as functions of temperature and wavelength, vol. 1. Humana Press, Totowa, NJ (1990)

Man, K.: Surface tension measurement of liquid metals by the quasicontainerless pendant drop method. Int. J. Thermophys. 21, 793 (2000)

Matsumoto, T., Fujii, H., Ueda, T., Kamai, M., Nogi, K.: Surface tension measurement of molten metal using a falling droplet in a short drop tube. Trans. JWRI. 34, 29 (2005)

Nowak, R., Lanata, T., Sobczak, N., Ricci, E., Giuranno, D., Novakovic, R., Holland-Moritz, D., Egry, I.: Surface tension of $\gamma$-tial-based alloys. J. Mater. Sci. 45, 1993 (2010)

Paradis, P.F., Ishikawa, T., Yoda, S.: Non-contact measurements of surface tension and viscosity of Niobium, Zirconium, and Titanium 
using an electrostatic levitation furnace. Int. J. Thermophys. 23, 825 (2001)

Rhim, W.K., Ohsaka, K., Paradis, P.F., Spjut, R.E.: Noncontact technique for measuring surface tension and viscosity of molten materials using high temperature electrostatic levitation. Rev. Sci. Instrum. 70, 2796 (1999)

Ricci, E., Novakovic, R.: Wetting and surface tension measurements on gold alloys. Gold Bull. 34, 41 (2001)

Sageman, D.: Surface tension of molten metals using the sessile drop method. PhD thesis, Iowa State University, (1972)

Sauerland, S., Lohöfer, G., Egry, I.: Surface tension measurements on levitated liquid metal drops. J. Non-Cryst. Solids 156-158, 833 (1993)

Seidel, A., Soellner, W., Stenzel, C.: EML - an electromagnetic levitator for the International Space Station. J. Phys. Conf. Ser, 327. (2011)

Simon, M.: Recherches sur la capillarité. Ann. Chim. Phys. 32, 5 (1851)
Stauffer, C.: The measurement of surface tension by the pendant drop technique. J. Phys. Chem. 69, 1933 (1965)

Vinet, B., Garandet, J., Cortella, L.: Surface tension measurements of refractory liquid metals by the pendant drop method under ultrahigh vacuum conditions: Extension and comments on tate's law. J. Appl. Phys. 73, 3830 (1993)

William, S.J.: VI. On the capillary phenomena of jets. Proc. R. Soc. Lond. 29, 71 (1997)

Xue, L., Keblinski, P., Phillpot, S.R., Choi, S.U.-S., Eastman, J.A.: Two regimes of thermal resistance at a liquid-solid interface. J. Chem. Phys. 118, 337 (2003)

Publisher's Note Springer Nature remains neutral with regard to jurisdictional claims in published maps and institutional affiliations. 